\title{
In vivo spike-timing-dependent plasticity in the optic tectum of Xenopus laevis
}

\author{
Blake A. Richards' ${ }^{1}$ Carlos D. Aizenman ${ }^{2}$ and Colin J. Akerman ${ }^{1 *}$ \\ Department of Pharmacology, University of Oxford, Oxford, UK \\ 2Department of Neuroscience, Brown University, Providence, RI, USA
}

Edited by:

Per Jesper Sjöström, University College London, UK

Reviewed by:

Melanie A. Woodin, University of Toronto, Canada

Edward S. Ruthazer, Montreal

Neurological Institute, Canada;

Florian Engert, Harvard University, USA

*Correspondence:

Colin J. Akerman, Department of Pharmacology, University of Oxford,

Mansfield Road, Oxford, OX1 3QT, UK.

e-mail: colin.akerman@pharm.ox.ac.uk
Spike-timing-dependent plasticity (STDP) is found in vivo in a variety of systems and species, but the first demonstrations of in vivo STDP were carried out in the optic tectum of Xenopus laevis embryos. Since then, the optic tectum has served as an excellent experimental model for studying STDP in sensory systems, allowing researchers to probe the developmental consequences of this form of synaptic plasticity during early development. In this review, we will describe what is known about the role of STDP in shaping feed-forward and recurrent circuits in the optic tectum with a focus on the functional implications for vision. We will discuss both the similarities and differences between the optic tectum and mammalian sensory systems that are relevant to STDP. Finally, we will highlight the unique properties of the embryonic tectum that make it an important system for researchers who are interested in how STDP contributes to activity-dependent development of sensory computations.

Keywords: spike-timing-dependent plasticity, visual system, synaptic development, optic tectum, receptive field

\section{INTRODUCTION}

How do animals survive in ever-changing, complex environments? A critical feature is the brain's ability to exhibit lasting changes in neural circuits that enable flexible development, adaptation to changes in the environment, and storage of new information. The widespread consensus today is that these capabilities are mediated in part by long-term potentiation and depression (LTP/LTD) of synaptic efficacy (Martin et al., 2000), which are in turn driven by correlations in spiking activity (Bi and Poo, 2001). Uncovering the rules that determine how synapses change based on spiking activity was significantly advanced by the discovery that the temporal order of inputs to the hippocampus determines whether potentiation or depression is induced (Levy and Steward, 1983). A series of studies more than a decade later showed that synaptic plasticity in several systems depends on the specific timing of action potentials in presynaptic and postsynaptic cells (Markram et al., 1997; Debanne et al., 1998; Zhang et al., 1998; Sjöström et al., 2001; Tzounopoulos et al., 2004), a phenomena referred to as spike-timing-dependent plasticity (STDP). A full appreciation of how STDP is involved in development, adaptation, and information storage requires studies of STDP that are carried out in vivo. The first demonstration of in vivo STDP was performed in the embryonic optic tectum of the Xenopus laevis frog (Zhang et al., 1998). Since that initial study the optic tectum has continued to be a fruitful system for studying the roles played by STDP in the development and adaptation of sensory systems (Engert et al., 2002; Mu and Poo, 2006; Vislay-Meltzer et al., 2006; Pratt et al., 2008).

Research in the optic tectum has provided substantial insight into how STDP affects the function and organization of young, rapidly changing neural circuits in vivo. The evidence suggests that it plays a role in the development of fundamental properties of sensory circuits, such as receptive field (RF) architecture (Tao and
Poo, 2005; Vislay-Meltzer et al., 2006; Dong et al., 2009), recurrent excitation (Pratt et al., 2008), and computations regarding motion in the visual environment (Engert et al., 2002; Mu and Poo, 2006). There remain many questions regarding STDP, for which the optic tectum remains an ideal experimental system for investigation. These include how the STDP rule changes during the development of distinct cellular morphologies and layers, the role of different neurotransmitters in shaping the effects of STDP, and how STDP operates on a population level. Understanding STDP's place in this circuit is likely to continue to provide important clues regarding the developmental role of this form of plasticity across neural systems.

Despite its importance in our current understanding of synaptic plasticity, some researchers may be less familiar with the optic tectum than with other model systems. Long before the identification of STDP, the optic tectum was one of the systems of choice for studying the role of activity in neural development. For many decades researchers have used its unique properties to investigate how activity shapes properties such as topographic maps and binocular circuits. Therefore, before focusing upon more recent research on STDP, this review will begin with a brief overview of the optic tectum, its development and key contributions this system has made to our understanding of activitydependent mechanisms in development. A full discussion of the extensive research in these areas is obviously not possible here and we refer interested readers to the thorough reviews provided by others (Schmidt, 1985; Udin, 1985, 2007; Cline, 1991, 2003; Holt and Harris, 1993; Debski and Cline, 2002; Ruthazer and Cline, 2004). With this background in place, we will then review in vivo studies on STDP in the optic tectum, with the ultimate goal of linking this to the earlier work, thereby identifying future directions for STDP research that will be particularly tractable in this system. 


\section{BACKGROUND: THE OPTIC TECTUM AND ITS ACTIVITY- DEPENDENT DEVELOPMENT}

The optic tectum is a layered, dorsal midbrain structure (Figure 1A), which is referred to as the superior colliculus in mammals (Butler and Hodos, 2005). Based on Golgi stain studies, the optic tectum of amphibians is typically described as having 9 layers, and 14 distinct cellular morphologies (Figure 1B) (Székely and Lázár, 1967, 1976; Lázár, 1973). Compared to many systems, the optic tectum exhibits a great deal of anatomical and functional conservation across the phylogenetic tree (Ingle, 1973a; Butler and Hodos, 2005). The common traits of the optic tectum of all species are (1) it receives direct projections from the contralateral retina in its superficial layers, (2) it receives projections from other sensory modalities in deeper layers, (3) it sends most of its outputs directly to motor systems in the brainstem and spinal cord but also projects reciprocally with itself, the telencephalon, and the diencephalon, (4) its inputs and outputs are organized into topographic maps that are matched across sensory modalities (Butler and Hodos, 2005). Functionally, the optic tectum is important for spatial orienting behaviors in many animals, and neurons in the adult optic tectum of every species studied are sensitive to sudden, sharp movements, or local brightness fluctuations in the visual field (Ingle, 1973a). The optic tectum has a particularly important role in amphibians, as ablation of this structure renders adult frogs completely unresponsive to moving stimuli, including both potential prey and predators (Ingle, 1973b). Thus, the optic tectum is central to amphibians' visual processing capabilities (Grüsser and GrüsserCornehls, 1976).

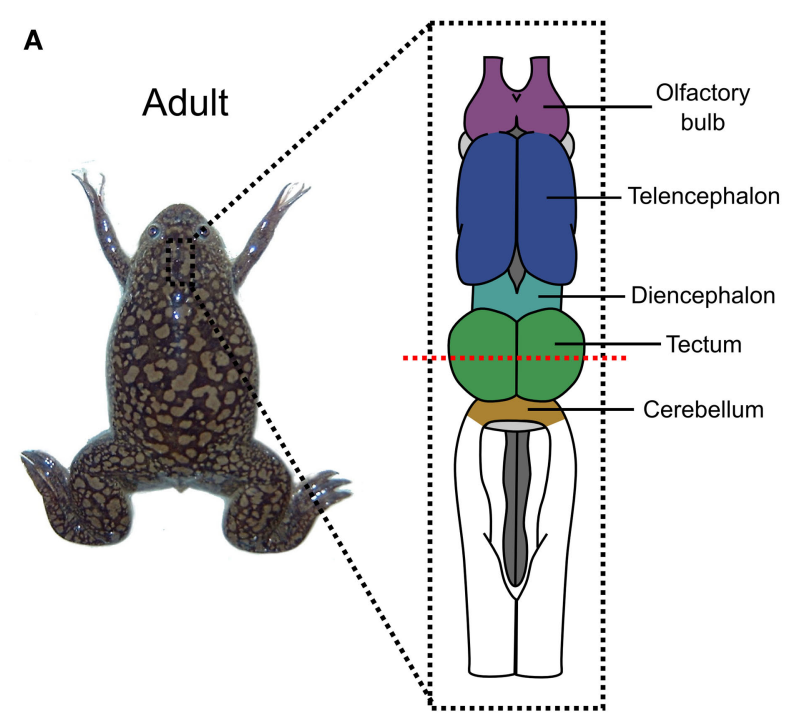

B

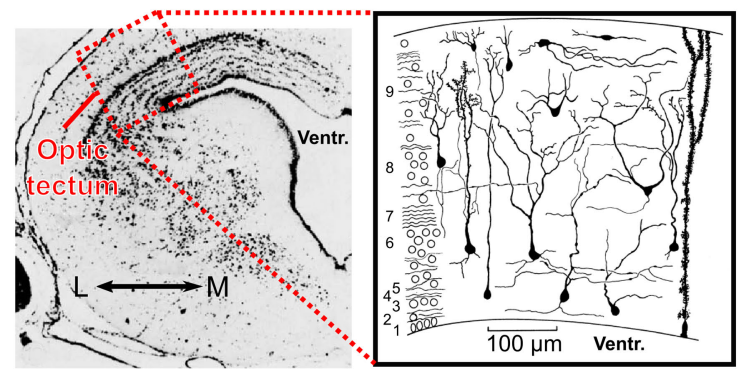

C
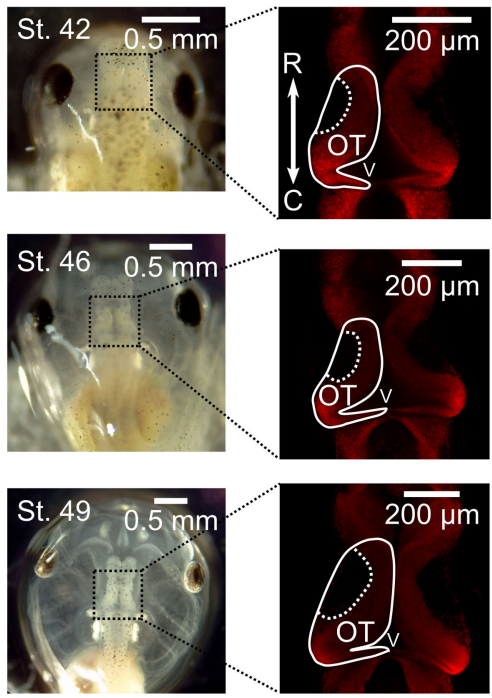

D

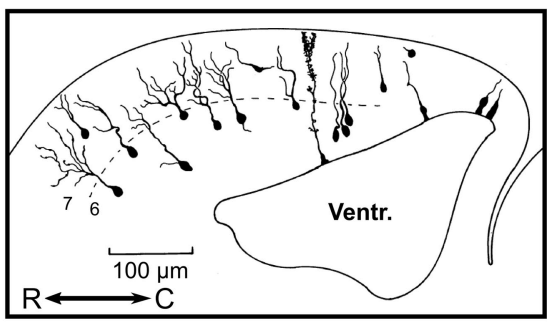

FIGURE 1 |Anatomy of the optic tectum in the adult Xenopus laevis and during tadpole development. (A) In the adult Xenopus laevis frog (photograph) the central nervous system (drawing) contains several distinct structures and the optic tectum is the roof of the large midbrain structure, situated caudally to the diencephalon and rostrally to the cerebellum. It is one of the largest dorsal structures in the Xenopus brain, along with the telencephalon and the olfactory bulb. (B) The layered structure of the adult optic tectum can be seen in the coronal section (left), which has been stained with cresyl-violet. The section is taken from the plane indicated by the red dashed line in (A). As the drawing illustrates (right), there are several distinct cellular morphologies found within the optic tectum, which have been classified into 14 categories. The numbers at the side of the drawing indicate the 9 different layers of the tectum. (C) Photographs (left) of Xenopus laevis tadpoles taken dorsally at stages 42,46 , and 49 illustrate the changes that occur during the stages in which STDP is typically studied. During this time the optic tectum grows, as shown by confocal images of whole-mount brains with propidium iodide staining for cell nuclei. The images shown are in the horizontal plane and at a depth of $100 \mu \mathrm{m}$ from the dorsal surface of the brain (right). Note the dark regions in the rostral-lateral optic tectum which are comprised mostly of neuropil. Neurogenesis takes place in the caudal-medial region surrounding the ventricle. (D) Due to the location of the neurogenerative zone there is a progression in the maturity and morphological complexity of cells in the optic tectum at these ages in the caudal-rostral axis, as shown by this camera lucida drawing of a sagittal slice from a stage 49 tadpole. Images in (B) and (D) are reproduced with permission from Lázár (1973) and Nikundiwe and Nieuwenhuys (1983). 
In the frog species $X$. laevis, axons from retinal ganglion cells (RGCs) first innervate the embryonic optic tectum within 2-5 days post fertilization (d.p.f.), depending on the temperature of the environment, and tectal neurons become visually responsive in the following hours (Holt and Harris, 1983; Holt, 1984). These ages are usually referred to as stages 37-39 according to the common staging system used (Nieuwkoop and Faber, 1967). Most of the research on synaptic plasticity in the Xenopus optic tectum has been carried out over the course of the subsequent stages of development (40-48) (Zhang et al., 1998, 2000; Tao et al., 2001; Engert et al., 2002; Tao and Poo, 2005; Lien et al., 2006; Mu and Poo, 2006; Vislay-Meltzer et al., 2006; Liu et al., 2007; Pratt et al., 2008; Dong et al., 2009), during which the number of cells increases substantially (Figure 1C) and the cells themselves develop more complicated morphologies (Figure 1D) (Cline et al., 1996). These stages are intriguing because they provide a window into the very earliest moments of the impact of retinal activity and visual experience on this system. Interestingly, tectal growth occurs solely in a caudal-medial zone while the retina grows concentrically (Gaze et al., 1979) and the repercussion for the system is that connections must migrate as the retinotopic map constantly shifts (Cline, 1991). Thus, during the stages of development in which STDP is actively shaping the tectal circuit, the structure is undergoing substantial changes while also serving as a functional sensory system.

The receptors and intrinsic channels of neurons in the optic tectum are very similar to other neural systems. Analysis of the intrinsic properties of tectal neurons in early life shows they possess voltage-gated tetrodotoxin (TTX) sensitive $\mathrm{Na}^{+}$currents, transient and steady-state $\mathrm{K}^{+}$currents, and a steady-state $\mathrm{Ca}^{2+}$ current (Aizenman et al., 2003). Monosynaptic retinotectal projections are glutamatergic, activating both $\alpha$-amino-3-hydroxy-5-methyl4-isoxazole propionic acid (AMPA) and $N$-methyl D-aspartic acid (NMDA) receptors (Hickmott and Constantine-Paton, 1993). Projections within the optic tectum can be either glutamatergic, mediated via AMPA and NMDA receptors, or $\gamma$-amino-butyricacid (GABA)-ergic, mediated by GABA-A receptors (Hickmott and Constantine-Paton, 1993). The AMPA receptors of the optic tectum are also known to include $\mathrm{Ca}^{2+}$-permeable AMPA receptors (Aizenman et al., 2002). All of these signals are present in X. laevis at early stage of life following the initial formation of retinotectal synapses (Zhang et al., 1998, 2000; Aizenman et al., 2002, 2003; Akerman and Cline, 2006). On top of these basic signals, the optic tectum at later stages of development is known to receive modulatory cholinergic inputs from the nucleus isthmus (Gernert and Ewert, 1995; Edwards and Cline, 1999; Dudkin and Gruberg, 2003). At these stages the isthmus conveys signals from one tectal lobe to the other, thereby providing the circuit with binocular information (Udin and Fisher, 1985). In summary, the monosynaptic, polysynaptic, and modulatory input properties are very similar to those found in the superior colliculus of mammals (Isa, 2002).

As mentioned above, the inputs to the optic tectum arrive in a topographically ordered map so that particular anatomical regions of the tectum are sensitive to particular areas of sensory space (Gaze, 1958). Seminal early research showed that surgical rotation of the eye in frogs led to altered behaviors due to a corresponding rotation of the retinotopic map in the contralateral tectum (Sperry, 1944; Gaze, 1959), an effect which was not found to be sensitive to visual experience during formation of the projections (Jacobson, 1968). This suggested that the retinotopic map may be determined solely by activity independent chemical cues (Sperry, 1963), a hypothesis which received later support from work demonstrating the importance of various chemical gradients in the tectum for guiding RGC axon growth (Cheng et al., 1995; Drescher et al., 1995; Mann et al., 2002). However, the reality has turned out to be that both the establishment and maintenance of retinotopic maps in the tectum involve an interplay of chemical cues and activity (Cline, 1991, 2003; Debski and Cline, 2002).

Researchers discovered that the initial retinotopic projections are largely overlapping and sort out over time (Gaze et al., 1974; O’Rourke and Fraser, 1986). Evidence suggests that this process is activitydependent, as TTX or the NMDA receptor antagonist 2-amino-5 -phosphonopentanoic acid (AP5) disrupt retinotectal refinement (Meyer, 1983; Schmidt and Edwards, 1983; Cline and ConstantinePaton, 1989). In addition, studies found that even a coarse retinotopic order in ipsilateral inputs, which arrive through an indirect route via the nucleus isthmus, is activity-dependent (Udin, 2007). Rotation of the ipsilateral eye produces an initially rotated ipsilateral retinotopic map, but with time the nucleus isthmus projections reorient themselves to match the contralateral inputs (Udin and Keating, 1981) in a process that requires visual experience (Keating and Feldman, 1975; Udin and Keating, 1981). The importance of activity has also been demonstrated through the use of surgically implanted third eyes onto frogs, which successfully innervate the optic tectum, but form segregated bands that are distinct from those of the native eye (Constantine-Paton and Law, 1978). This segregation is blocked both with TTX and NMDA receptor antagonists (Reh and ConstantinePaton, 1985; Cline et al., 1987). Studies suggest that these dynamic organizational properties in the tectum are mediated at least in part by activity-dependent growth and retraction of axonal and dendritic processes (Reh and Constantine-Paton, 1984; Cline and ConstantinePaton, 1990; O’Rourke et al., 1994; Rajan and Cline, 1998).

Early models put forward the idea that the strengthening or stabilization of mutually correlated inputs and weakening or retraction of uncorrelated inputs could help explain the observations of map remodeling within the retinotectal system (Changeux and Danchin, 1976; Willshaw and Von Der Malsburg, 1976; Whitelaw and Cowan, 1981). The necessity of visual experience, spiking activity, and NMDA receptor activation for organizing tectal inputs suggests that something akin to Hebbian LTP may be at work in the developing optic tectum (Cline, 1991; Ruthazer and Cline, 2004). Indeed, evidence from in vivo imaging suggests that NMDA receptors function as a correlation detecting mechanism for stabilizing or retracting connections (Ruthazer et al., 2003). It is possible that STDP itself interacts with these mechanisms, or even underpins some of them (Udin, 2007).

\section{FIRST DEMONSTRATION OF STDP IN THE OPTIC TECTUM}

The first demonstration of STDP in vivo was the seminal 1998 study by Zhang et al. (1998). The implications of this study were relevant to systems other than the optic tectum, but the fact that retinotectal map refinement is activity-dependent (Cline and ConstantinePaton, 1989), also made it directly relevant to the previous work that had been carried out in this system. The authors took advantage of the unrefined state of the retinotectal projections in embryonic 
Xenopus. RGC axon arbors at stages 40-41 cover a substantial area of the tectum, such that a single tectal cell receives inputs from many different RGCs at many different positions across the retina. Zhang et al. (1998) used this feature to examine how inputs from two different RGCs might compete or co-operate in the induction of synaptic plasticity on the same tectal cell. Whole-cell perforated patch recordings of single tectal neurons were performed concurrently with loose-patch stimulation of two different RGCs that formed excitatory synaptic connections onto the tectal neuron (Figure 2A). Consistent with previous work on the mechanisms of LTP induction (Martin et al., 2000), Zhang et al. (1998) found

A

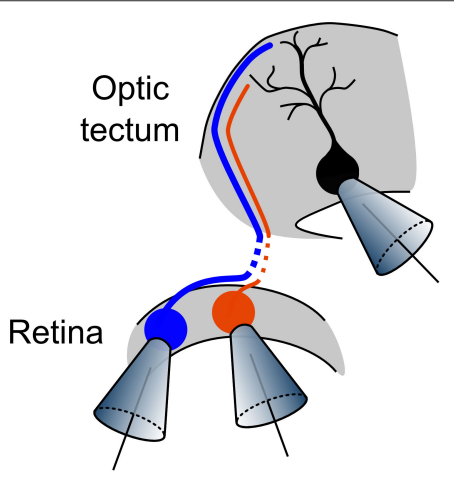

B

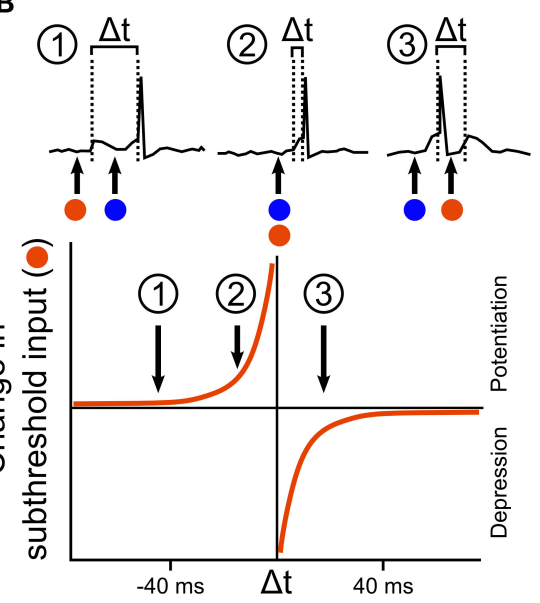

FIGURE 2 | First in vivo observation of STDP in the optic tectum. (A) The first in vivo demonstration of STDP was performed by Zhang et al. (1998) in an elegant experiment, illustrated here. Activity from a single tectal neuron was recorded using whole-cell perforated patch, while two different RGCs that form synaptic connections onto the tectal neuron were loose-patched, allowing stimulation for induction of LTP or LTD. In some of the recordings, one RGC produced suprathreshold responses (illustrated by the blue cell), whilst the other produced only subthreshold responses (illustrated by the orange cell). (B) Analysis of the effects of the timing of the inputs showed that the effect of stimulation on the subthreshold input depended on the timing of its excitatory postsynaptic potentials (EPSPs) relative to the tectal spike that was triggered by the suprathreshold input. If the subthreshold EPSP preceded the tectal spike by more than $20 \mathrm{~ms}$, the input was relatively unaffected (inset 1). However, if the subthreshold EPSP occurred within the $20 \mathrm{~ms}$ before the suprathreshold EPSP, and therefore just before the tectal spike, the input was strongly potentiated (inset 2). In stark contrast, if the subthreshold EPSP occurred during the $20 \mathrm{~ms}$ immediately following the tectal cell spike, this input was depressed (inset 3). Examination of the effects of a range of timing differences led to an estimated curve for the STDP rule in retinotectal synapses. that repetitive stimulation of a single RGC could induce potentiation of AMPA mediated excitatory postsynaptic currents (EPSCs), but only for the stimulated input and only if the tectal cell spiked and NMDA receptors were active. They also found that if the two inputs were activated simultaneously, and co-operated to induce spiking, they were both strengthened. The exciting discovery Zhang et al. (1998) made was that if the RGCs were repetitively stimulated at different times, the relative timing of the two inputs determined the effect on the synaptic connections. If the tectal cell spiked after the second input, both connections were strengthened, though the second connection was potentiated more than the first. In contrast, if the tectal cell spiked after the first input but before the second, the first connection was strengthened whereas the second was actually weakened. Zhang et al. (1998) performed a careful analysis of the relationship between the changes in synaptic strength and the timing of the RGC and tectal cell spikes, which revealed the now well-known asymmetric exponential relationship that characterizes STDP in a number of systems (Figure 2B) (Dan and Poo, 2004).

The implications of these findings for development of the optic tectum were intriguing. Since spiking of tectal cells was required for changes in synaptic strength, it suggested that a sub-threshold input would either be co-operatively enhanced or competitively eliminated by a different, supra-threshold input, in a manner that critically depended on their relative timing. As such, early relationships in spike-timing between cells on the order of milliseconds are not inconsequential, and might be important determinants of the functional maturation of the system. This might be one of the mechanisms by which activity directs the development of topographic maps in the optic tectum - a possibility that has yet to be fully addressed. More broadly, another implication was that in vivo STDP could be one mechanism by which the environment exerts an instructive influence on the development of visual systems, as different statistics in the visual environment would produce different patterns of spike-timing. A later paper by the same authors demonstrated that visual inputs could also induce LTP in retinotectal connections (Zhang et al., 2000), an important demonstration if STDP were to actually enable an instructive role for the environment in optic tectum development. However, the exact functional consequences, and how the timing requirements of STDP might affect the emergence of computational properties of the neural circuits, remained to be established. Over the course of the decade following Zhang et al.'s (1998) study, this became one of the central themes of research on STDP in the Xenopus optic tectum.

\section{STDP AND THE DEVELOPMENT OF DIRECTION SELECTIVITY}

A comprehensive analysis of responses in the adult optic tectum of $X$. laevis has not been conducted, but it is known that in other frog species neurons in the adult optic tectum show a variety of response profiles, many of which exhibit direction selectivity - i.e., a bias in a cell's response to stimulus movement in a particular direction (Grüsser and Grüsser-Cornehls, 1976). How this property might emerge from visual experience was illustrated by Engert et al. (2002) in a study on instructive learning in Xenopus tadpoles of stages 42-45. They presented tadpoles with visual stimuli by focusing an image of an LCD screen onto their retinas. Repetitive presentation of a white bar moving across a dark background produced an increase in the synaptic currents tectal neurons received during presentation 
of the bar (as measured by whole-cell perforated patch-clamp), but only for the direction that had been presented - other directions of movement showed no potentiation. Engert et al.'s data suggested that this was due to an asymmetric alteration of the tectal cells' excitatory RFs, such that those areas of the RF that were active early in the presentation of the bar were potentiated. Computational studies have suggested that asymmetric RF alterations of this sort could be produced by STDP (Mehta et al., 2000; Shon et al., 2004) and, given that the changes required postsynaptic spiking activity, it was proposed that the stimulus-driven learning observed in the tectum might be mediated by STDP (Engert et al., 2002).

Evidence supporting this postulate was provided in a later study by $\mathrm{Mu}$ and Poo (2006). After verifying the observations of Engert et al., the authors utilized a modified experimental protocol to demonstrate how STDP could produce the result. Rather than presenting a continuously moving bar, $\mathrm{Mu}$ and Poo presented the tadpoles with a bar in three different positions, flashed sequentially to mimic movement across the retina (Figure 3A). They then injected currents into the soma of the tectal cells to control when the cells spiked relative to the presentation of the three bars. As one would predict from the STDP rule previously observed in the optic tectum (Zhang et al., 1998), they found that there was a different effect on the excitatory RF depending on when the bar flash occurred relative to the tectal spikes: areas of the RF where bars had flashed before the spikes showed potentiated connections to the tectal cells, whereas areas where the bar had flashed after the spikes showed depressed connections. This supported the conclusion that asymmetric RF changes associated with exposure to a moving bar (Engert et al., 2002) could indeed be mediated by STDP. The study by Mu and Poo (2006) also highlighted candidate cellular mechanisms underlying STDP in the optic tectum. The asymmetric changes in excitatory RFs were altered both by inhibition of brain-derived neurotrophic factor (BDNF) signaling and nitric oxide (NO) synthase, but in different ways: inhibition of BDNF-trkB signaling eliminated the potentiation in the RFs, whereas inhibition of NO synthase eliminated the depression. This demonstrated a functional dissociation according to both the signaling mechanisms and the functional consequences of spike-timing-dependent potentiation and depression.

These studies (Zhang et al., 1998; Engert et al., 2002) provided some of the first in vivo experimental evidence that asymmetric RF alterations induced by STDP could produce direction selectivity, verifying predictions of computational models (Figure 3B) (Mehta et al., 2000; Rao and Sejnowski, 2001; Shon et al., 2004). It is interesting to note that more recent studies in mammalian primary visual cortex suggest that the development of direction selective neurons

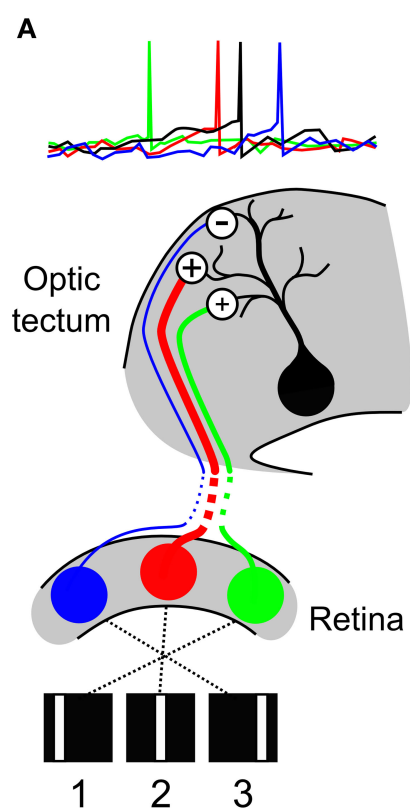

FIGURE 3 | Development of direction selectivity and RF structure via STDP in the optic tectum. (A) The principle of how STDP can induce direction selectivity in the optic tectum was demonstrated by Mu and Poo (2006), by mimicking movement across the retina with flashes of a white bar at three different locations in visual space. If the tectal cell (black) was forced to spike soon after the second flash, the RF of the cell was altered by STDP in an asymmetric manner that potentiated responses to the first and second bars (green and red cells), but depressed responses to the third bar (blue cell). (B) Asymmetric changes in a RF can produce direction selectivity due to the differences in temporal summation for one direction versus the other. If the strengthened inputs are activated first, they can summate with subsequent inputs to produce a high level of depolarization in postsynaptic tectal cells

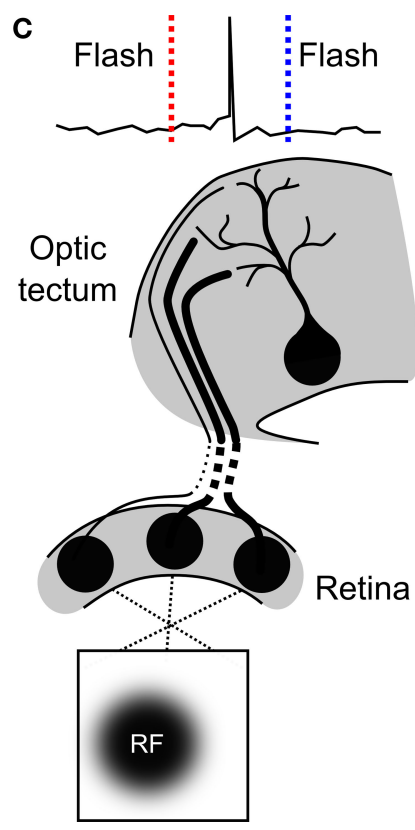

D

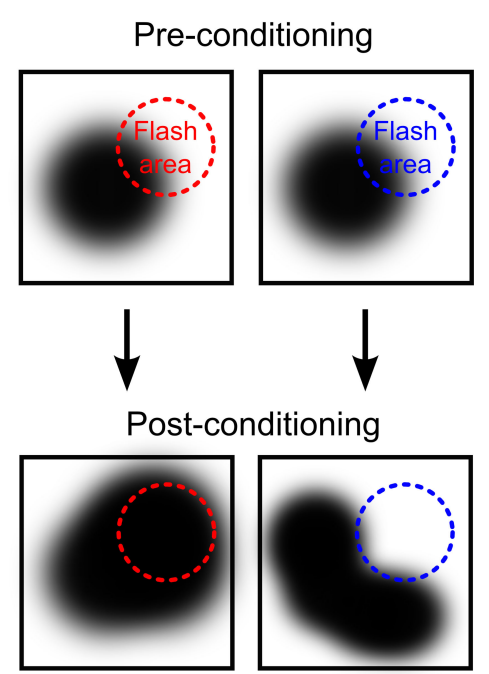

producing suprathreshold activity (top, dashed line indicates hypothetical spike threshold). In contrast, if the weaker inputs are stimulated first, they will have decayed by the time subsequent inputs arrive and so less temporal summation occurs and inputs remain subthreshold (bottom). (C) The strength of the connections onto a tectal neuron determines its RF profile, as illustrated here for a hypothetical cell. (D) Vislay-Meltzer et al. (2006) demonstrated that this RF profile could be altered by STDP to either move towards or away from a given region of space. If a flash occurred prior to a tectal cell's spikes (red line), the RF tended to shift toward that area. In contrast, if a flash occurred after a tectal cell's spikes (blue line) the RF tended to shift away from the area of the flash. Interestingly, they also observed that the RFs potentiated in areas outside of the area of the flash, as shown. 
is very sensitive to the statistics of the early environment (Li et al., 2006, 2008), indicating that visually driven STDP may be a general mechanism for establishing direction selectivity.

\section{STDP AND RF DEVELOPMENT IN THE OPTIC TECTUM}

Early electrophysiology experiments examining multi-unit RFs in tadpoles suggested that RFs in young animals (before stage 47) are very large, but grow smaller over development (Gaze et al., 1974). This observation has been confirmed by more recent experiments using whole-cell voltage clamp recordings. Over stages 43-48, the visuotopic mapping of excitatory synaptic inputs to individual tectal cells reveal that their RF shrinks and becomes sharper with age, and that this process is NMDA receptor and GABA-A receptor dependent (Tao and Poo, 2005; Dong et al., 2009). This shrinking of the excitatory RF is coupled with increased spatial alignment between excitatory synaptic inputs, and GABAergic inhibitory synaptic inputs (Tao and Poo, 2005). These refinements appear to have behavioral implications, because even at these early stages of life RF refinement is required for the acquisition of a motion avoidance behavior in tadpoles (Dong et al., 2009).

What are the synaptic mechanisms behind such developmental refinements in RFs? One factor that would influence excitatory RF size is the distribution of synaptic inputs as constrained by the spatial extent of RGC axon arbors, relative to the size of the growing tectum (Sakaguchi and Murphey, 1985). However, this may not explain the requirement for NMDA and GABA-A receptor signaling in RF refinement. An alternative possibility is that RF refinement is directly related to STDP. In a 2006 study, Vislay-Meltzer et al. (2006) examined exactly how STDP affects the structure of excitatory RFs of tadpoles stage 41-45. In this study whole-cell perforated patch-clamp recordings were used to map excitatory RFs with a reverse-correlation technique. Then the cells experienced a training period during which a stimulus was repeatedly flashed in a restricted area of visual space whilst the experimenter controlled the spiking behavior of the tectal cell. After this the RF was remapped and the effects of the training period were examined. In accordance with an STDP rule in the optic tectum at these ages, they found that if the tectal cells spiked immediately after the flash, that area of the RF was potentiated, whereas if the cells spiked immediately before the flash, that area of the RF was depressed (Figures 3C and 3D). This demonstrated, importantly, that STDP could affect changes in the structure of RFs in a manner that depends upon both the spatiotemporal statistics of the sensory stimuli and the neural activity patterns.

In addition to the observation that STDP can shape tectal RF structure, the authors observed some very interesting changes that one might not have predicted. Not only did changes occur in those areas of the RF where the flashes had occurred, but also outside of these areas: in the case of either potentiation or depression in the trained region of the RF, the rest of the RF showed a net potentiation. Because this did not occur when the cells were clamped at hyperpolarized membrane potentials, it suggested that the effect was not due to plasticity occurring at synapses onto other cells. One explanation of these results is that the calcium signals that are important for STDP (Dan and Poo, 2004) might have spread to other areas of the cells' dendrites. This hypothesis is supported by the separate observation that immature tectal neurons show heterosynaptic plasticity effects mediated by calcium signals (Tao et al., 2001). However, this form of heterosynaptic spread of potentiation was induced with a theta-burst stimulation protocol and it is not known whether visually driven activity patterns could trigger heterosynaptic spread of potentiation. An alternative explanation is that potentiation also occurs within recurrent circuits of the tectum - at glutamatergic synapses from other tectal cells that converge onto the recorded neuron. At this point, this hypothesis cannot be fully assessed, as the interactions between STDP and recurrent circuitry in the optic tectum are only just beginning to be understood.

\section{STDP AND DEVELOPMENT OF RECURRENT TECTAL CIRCUITS}

The tectum is known to possess recurrent excitatory circuitry (Székely and Lázár, 1976; Hickmott and Constantine-Paton, 1993; Nakagawa et al., 1997). Recurrent connections can provide critical abilities for neural circuits, enabling persistent activity for a wider range of prediction and information storage capabilities (McCormick et al., 2003), as well as helping networks to maintain dynamics that increase general computational power (Bertschinger and Natschläger, 2004; Maass and Markram, 2004). Their contributions to the function of the optic tectum in amphibians are not fully known, but it is thought that they help to integrate information across modalities, and thereby aid in prey catching and predator avoidance behaviors (Grüsser and Grüsser-Cornehls, 1976). In mammals, recurrent excitation in the superior colliculus enables bursting activity that is important for direction of gaze-orienting movements (Sparks, 1986; Lee et al., 1997; Saito and Isa, 2003).

In a 2008 study, Pratt et al. (2008) examined the development of recurrent excitatory connections in the optic tectum of tadpoles between stages $44-49$. First, they observed that in young animals (stages 44-46) stimulation of the optic nerve produced prolonged spiking, consistent with a recurrent excitation feed-back loop. Whole-cell recordings revealed excitatory synaptic currents that were not eliminated by physically isolating the tectum from other parts of the brain, suggesting that the prolonged tectal cell spiking was sustained by intratectal AMPA receptor mediated inputs. Interestingly, Pratt et al. (2008) observed that the temporal profiles of both the spike-trains and the excitatory currents were both significantly different in stage 49 animals, showing a tendency towards an increase in the early responses and a decrease in the later responses. Trial to trial spike time variability was also reduced in the older animals. This suggested that over these developmental stages the recurrent circuitry of the optic tectum is refined, such that the temporal processing of the retinal information is altered (Figure 4A).

Pratt et al. (2008) hypothesized that these changes may be mediated by STDP at intratectal synapses, such that when a presynaptic tectal cell tends to spike early synapses onto its postsynaptic partners would be strengthened by STDP, whilst tectal-tectal synapses where the presynaptic partner tends to spike late would be weakened. In this scenario STDP would reshape the circuit to favor synapses where the presynaptic partner gives rapid, reliable responses, thereby increasing the temporal precision of the recurrent excitation. As an initial assessment of this hypothesis they employed a training protocol of paired pulses to the optic nerve to mimic the hypothesized scenario. An initial pulse was used to stimulate the 

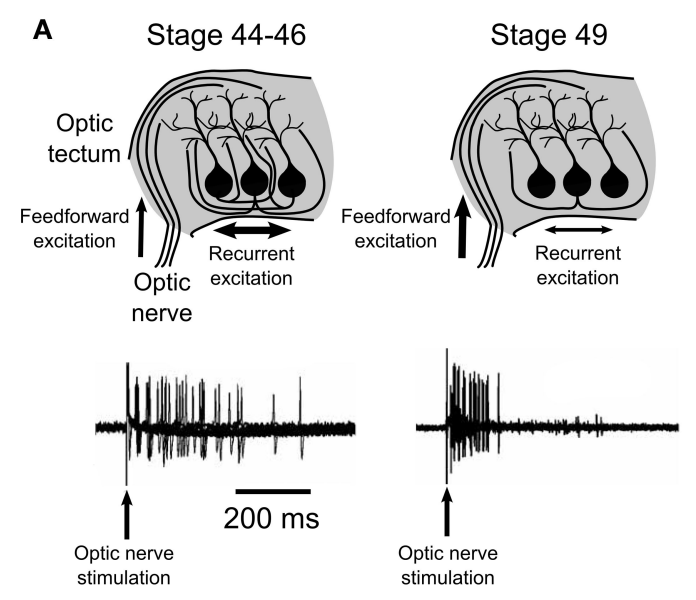

FIGURE 4 | Spike-timing-dependent plasticity and recurrent excitatory circuits in the optic tectum. (A) Pratt et al. (2008) observed evidence for a developmental refinement of the recurrent excitatory circuitry of the tectum between stages 44 and 49 . Stimulation of the optic nerve at early stages (44-46) produced prolonged spiking activity, while stimulation at a later stage (49) produced an initial strong response that did not show prolonged activity, as
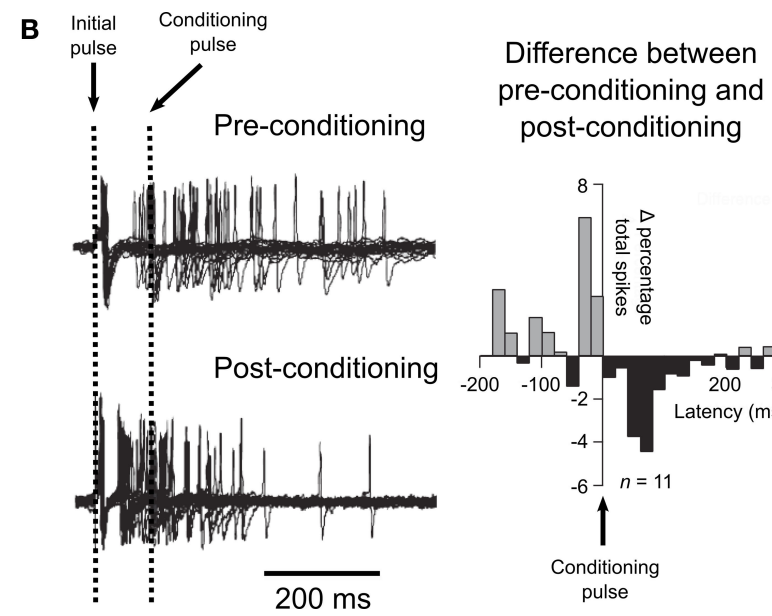

pre-conditioning and

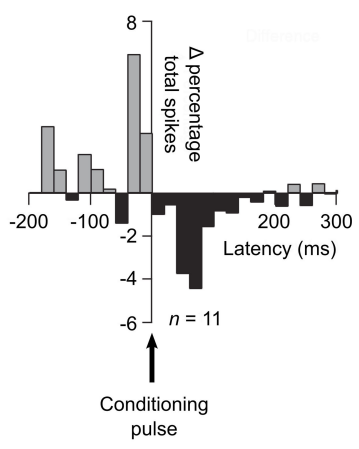

illustrated by traces from loose-patch recordings shown here. (B) Training young tectum by delivering a timed "conditioning" pulse to the optic nerve induced a similar reshaping of spiking to that observed over development. Examination of the differences in the percentage of spikes that occurred before or after the conditioning pulse showed an effect that supported a role for STDP in this refinement of recurrent circuitry. Data are reproduced from Pratt et al. (2008). recurrent excitation, while a second "conditioning" pulse was used to provoke STDP amongst tectal neurons. The authors predicted that any intratectal synapses where the presynaptic partner spiked before the conditioning pulse would show potentiation, whereas synapses where the presynaptic partner spiked after the conditioning pulse would depress. The post-conditioning temporal profiles of tectal spiking suggest that this is exactly what occurred (Figure 4B). In addition, the authors showed that this could take place in vivo via visual experience. Tadpoles raised in environments where paired flashes of light occurred at particular intervals showed temporal profiles of recurrent excitation that reflected the time between flashes. Taken together, this data suggests that in vivo STDP sculpts the intratectal recurrent excitatory drive in a manner that reflects the temporal properties of the animals' environments.

A question raised by Pratt et al.'s (2008) study is what is the functional purpose of this early reshaping of recurrent circuitry in the optic tectum? One possibility is that sharpening of the circuit is related to the spatial refinement of RFs in the system (Gaze et al., 1974; Tao and Poo, 2005), as much of the RF of tectal neurons may be mediated by the local circuit. Moreover, it seems possible that if the initial spatial RF requires refining, so too does the temporal RF. Alternatively, the increased precision may reflect a shift towards a neural code with greater temporal precision, which would require less integration time for accurate transmission of signals (VanRullen et al., 2005). Given the importance of the optic tectum for rapid detection of moving stimuli and orienting responses in frogs (Ingle, 1973b), it would be very interesting if one of the effects of STDP was to shift the circuit toward a "spike-timing code" that improved the rapidity with which the animals could respond to prey and predators. Equally, STDP in the recurrent circuits may be important for establishing proper multisensory integration within the tectum. Given the general importance of recurrent circuitry in many neural systems, the role and functional implications for STDP in shaping these connections deserves further investigation.

\section{CHANGES IN STDP DURING DEVELOPMENT OF THE OPTIC TECTUM}

The maturation of a circuit undoubtedly affects the rules for induction of synaptic plasticity. As we have seen, the optic tectum undergoes a number of dramatic changes in the days following innervation by RGC axons: retinotopic maps are readjusted and refined (Ruthazer and Cline, 2004), excitatory and inhibitory synaptic inputs are brought into spatial alignment (Tao and Poo, 2005), and recurrent circuitry is reshaped to favor greater precision in visual responses (Pratt et al., 2008). In addition to these adjustments at the systems-level, neurons in the optic tectum during this period are changing dramatically at a cellular-level. As the various distinct cellular morphologies emerge in the optic tectum (Lázár, 1973), the morphological complexity of tectal cells increases via a process that relies on both glutamatergic signaling and CaMKII (Rajan and Cline, 1998; Wu and Cline, 1998; Haas et al. 2006). Increased morphological complexity can alter synaptic plasticity by ensuring input specificity in LTP induction (Tao et al., 2001) and fundamental aspects of synaptic transmission are also altered during this period. The GABA-A receptor reversal potential in tectal neurons at early stages of life is depolarized relative to the resting membrane potential, which changes by stages $48-50$ and has implications for NMDA receptor activation (Akerman and Cline, 2006). Moreover, the ratio of the strength of glutamatergic to GABAergic inputs is altered during this period. Tectal neurons shift from a GABAergically dominated regime to one exhibiting greater balance, and interestingly, this ratio determines the nature of visually driven plasticity of GABAergic synapses in the system (Liu et al., 2007). On top of all these changes, tectal neurons alter their sodium channel activity in a manner that tends to stabilize their input-output functions (Pratt and Aizenman, 2007). Taken together, it seems plausible that during development the induction rules for LTP and LTD may change from the original STDP rule observed at stages $40-41$ by Zhang et al. (1998) (Figure 2B). This has been investigated by Tsui et al. (2010) in a study published in this issue. 
Using a whole-cell perforated patch-clamp experimental preparation Tsui et al. (2010) investigated STDP at different stages of tectal development. In agreement with previous work on retinotectal STDP in wild-type animals stages 41-44 (Zhang et al., 1998; Mu and Poo, 2006; Vislay-Meltzer et al., 2006), they observed that pairing a RGC input with a tectal spike produced either LTP or LTD dependent on the timing of the input relative to that of the spike. However, they found that the same protocols did not produce LTP or LTD in wild-type animals of stages 46-48. Nonetheless, they did observe that repetitive low-frequency stimulation could produce LTD at these later stages. This data demonstrates that the induction mechanisms for plasticity change during tectal development in a manner that affects STDP.

One interpretation of this data is that there is a critical developmental window for STDP to shape the optic tectum in Xenopus that lasts only for a few days following innervation by the retina, after which STDP no longer plays a part in the modification of this circuit. However, it is possible that synaptic plasticity at these stages could still be "timing-dependent," i.e., sensitive to the precise timing of presynaptic and postsynaptic action potentials, but as the animals age there may be additional requirements for the induction of synaptic changes. For example, it could be that multiple synaptic inputs must be activated co-operatively for the necessary molecular signals to be triggered. Alternatively, signals from other neural systems may be required to enhance the incoming inputs, similar to the manner in which cholinergic signals from the nucleus isthmus can enhance axonal calcium transients in RGC inputs (Edwards and Cline, 1999; Dudkin and Gruberg, 2003). Another consideration must be that GABAergic inputs are more hyperpolarizing at these older stages of development (Akerman and Cline, 2006), and it is possible that under these conditions of more hyperpolarizing GABA, bursts of postsynaptic spikes become necessary to trigger synaptic plasticity, as has been reported in other systems (Meredith et al., 2003).

\section{TOWARDS THE FUTURE OF STDP RESEARCH IN THE OPTIC TECTUM}

There are many unresolved questions surrounding STDP, and the optic tectum of $X$. laevis is an ideal system in which to address many of them. Almost any issue related to STDP could be investigated in the optic tectum, from the molecular mechanisms underlying STDP to its long-term behavioral consequences. Here we will concentrate on questions for which this experimental system is particularly well placed to provide answers, due to its specific physiological, functional, and developmental properties.

One important issue for future investigation is how the development of dendritic morphology in early life affects STDP. Several studies have suggested that the rules governing STDP are very different depending upon the location of synapses on dendrites and the active properties of dendrites themselves (Kampa et al., 2007). Within the optic tectum, it is known that dendritic complexity affects the input specificity of LTP induction (Tao et al., 2001). The various distinct cellular morphologies of the optic tectum begin to emerge during the same stages where STDP is known to occur, such that by stage 49 most of the morphological traits of the adult can be seen in a subset of cells in the optic tectum (Lázár, 1973). These stages also provide a unique potential for studying this issue due to the caudal-medial mode of growth of the system (Figure 2B).
Cells in the rostral part of the tectum are relatively more mature and show greater morphological complexity compared to those in the caudal part of the tectum (Figure 1D), thereby allowing researchers to examine cells of greatly different morphological class and maturity within the same animal (Rajan and Cline, 1998; Wu and Cline, 1998). Future work could examine how STDP induction at both retinotectal and intratectal synapses changes as a function of the morphological maturity of the cells. Equally, it will be important to establish how input specificity of plasticity is controlled during development, perhaps through changes in local intracellular signaling mechanisms or through visually driven neural-glia interactions in the tectum (Tao et al., 2001; Tremblay et al., 2009). It is also known that topographic mapping of retinal inputs is achieved at the level of individual dendrites of tectal cells (Bollmann and Engert, 2009). It would be interesting to investigate whether this subcellular mapping is achieved via STDP mechanisms.

One branch of STDP research that may prove critical for our understanding of the role of STDP in development is how developmental changes in GABAergic signaling impinge on this form of plasticity. GABAergic signals are known to modulate synaptic plasticity induction (Meredith et al., 2003), and evidence suggests they control a variety of activity-dependent plasticity mechanisms, possibly by modulating NMDA receptor transmission, controlling the statistics of spiking activity, or both (Akerman and Cline, 2007). Developmental shifts in GABAergic signals, such as changes in GABA-A receptor reversal potential, or alterations in inhibitory synaptic architecture and plasticity, may therefore have major ramifications for STDP induction. GABAergic transmission in the tectum has been shown to be important for the maturation of tectal dendritic arbors (Shen et al., 2009). Moreover, GABAergic inputs to tectal cells are plastic and are being actively reshaped by visual experience in a manner that is dependent on the developmental stage of the animal (Lien et al., 2006; Liu et al., 2007). Researchers can therefore use the optic tectum of Xenopus to address both how GABAergic signals may control STDP-mediated learning and how this might change over development.

Another area where STDP may be important is in the integration of multisensory information (Davison and Fregnac, 2006). The optic tectum is an obvious candidate for studying such questions due to its functional role in integrating cross-modal signals for initiating spatial orienting behaviors (Butler and Hodos, 2005). Indeed, studies have suggested that STDP could provide the superior colliculus with the ability to develop some of these capabilities (Huo et al., 2008; Yu et al., 2009). There is still much to learn about the development of multi-modal inputs in the optic tectum, but what is known is that axons from the hindbrain carrying information from mechanosensory neurons arrive in the optic tectum at the same time as retinal projections and converge on deeper layers of the tectum along more proximal dendritic processes than those that receive retinal inputs (Deeg et al., 2009; Hiramoto and Cline, 2009). Individual tectal neurons at these young ages receive glutamatergic inputs from both sensory modalities (Deeg et al., 2009; Hiramoto and Cline, 2009; Pratt and Aizenman, 2009) and show interesting developmental changes in their synaptic transmission properties (Deeg et al., 2009). Understanding how STDP might affect the integration of these different sensory modalities is yet to be examined. 
Finally, we would also like to draw attention to a possible future role for the optic tectum in understanding STDP at a systems-level. A complete understanding of how STDP affects neural circuits will require studies of population wide activity at a single-cell, singlespike resolution. This presents a technical challenge, but one that might be best met with optical imaging techniques (Scanziani and Hausser, 2009). The optic tectum of animals like X. laevis or the zebrafish provide researchers the opportunity to measure activity across a large segment of an entire sensory circuit in a non-invasive manner (Niell and Smith, 2005). Researchers have already used this system to study binocular plasticity (Ramdya and Engert, 2008), and metaplasticity rules (Dunfield and Haas, 2009) across hundreds of cells simultaneously in the fully intact tectal circuit. As the temporal resolution of imaging techniques improves, this approach may prove invaluable to understanding STDP in a larger, circuit-wide context. Issues such as how STDP shapes topographic maps, adjusts circuits during periods of growth, or induces population coding of multisensory information are all questions that could be directly addressed in the optic tectum given the right imaging techniques.

\section{CONCLUSION}

The importance of the embryonic optic tectum for research into STDP is largely based on the interesting developmental questions that this system poses. But it is also based on the fact that it is possible to perform careful, well-controlled and physiologically relevant in vivo studies in the optic tectum. Of course, a complete picture of STDP will only emerge with research being carried out in many different systems. And to that end, researchers have investigated the possible roles of STDP in several other sensory systems in vivo, including the primary visual cortex of rodents (Meliza and Dan, 2006), cats, and humans (Yao and Dan, 2001), the olfactory bulb of locusts (Cassenaer and Laurent, 2007), the auditory cortex of ferrets (Dahmen et al., 2008), and the barrel cortex of rodents (Celikel et al., 2004). Together, these studies suggest that STDP is a compo-

\section{REFERENCES}

Aizenman, C. D., Akerman, C. J., Jensen, K. R., and Cline, H. T. (2003). Visually driven regulation of intrinsic neuronal excitability improves stimulus detection in vivo. Neuron 39, 831-842.

Aizenman, C. D., Muñoz-E ias, G., and Cline, H. T. (2002). Visually driven modulation of glutamatergic synaptic transmission is mediated by the regulation of intracellular polyamines. Neuron 34, 623-634.

Akerman, C. J., and Cline, H. T. (2006). Depolarizing GABAergic conductances regulate the balance of excitation to inhibition in the developing retinotectal circuit in vivo. J. Neurosci. 26, 5117-5130.

Akerman, C. J., and Cline, H. T. (2007). Refining the roles of GABAergic signaling during neural circuit formation. Trends Neurosci. 30, 382-389.

Bertschinger,N., and Natschläger,T.(2004). Real-time computation at the edge of chaos in recurrent neural networks. Neural. Comput. 16, 1413-1436.
Bi, G., and Poo, M. (2001). Synaptic modification by correlated activity: Hebb's postulate revisited. Annu. Rev. Neurosci. 24, 139-166.

Bollmann, J. H., and Engert, F. (2009). Subcellular topography of visually driven dendritic activity in the vertebrate visual system. Neuron 61, 895-905.

Butler, A., and Hodos, W. (2005). Comparative Vertebrate Neuroanatomy: Evolution and Adaptation. Hoboken, NJ: John Wiley and Sons.

Cassenaer, S., and Laurent, G. (2007). Hebbian STDP in mushroom bodies facilitates the synchronous flow of olfactory information in locusts. Nature 448, 709-713.

Celikel, T., Szostak, V. A., and Feldman, D. E. (2004). Modulation of spike timing by sensory deprivation during induction of cortical map plasticity. Nat. Neurosci. 7, 534-541.

Changeux, J., and Danchin, A. (1976). Selective stabilisation of developing synapses as a mechanism for the speci-

nent of sensory circuit plasticity in many different contexts and that it may represent an early evolutionary adaptation of neural systems. To really flesh out such a high-level, phylogenetic conception of this issue though, requires a comparative approach that examines both the molecular underpinnings and functional consequences of STDP in all the systems in which it is found. The optic tectum presents neuroscientists with an excellent model for this endeavor, due to the similarity of its function and connectivity across species (Ingle, 1973a; Butler and Hodos, 2005). And, continued investigations into the relationship between STDP and the development of the optic tectum have the potential to bring our understanding of this plasticity phenomenon in-line with the large body of scholarly work on activity-dependent development in the retinotectal circuit. As the first system in which STDP was demonstrated in vivo, and as one of the early favored systems for studying activity-dependent development, the optic tectum will continue to be an important model for STDP research.

\section{ACKNOWLEDGMENTS}

We would like to thank Leah Herrgen for assistance in preparing the figures and Ed Ruthazer for his helpful comments on the manuscript. The research leading to these results has received funding from the European Research Council under the European Community's Seventh Framework Program (FP7/2007-2013), ERC grant agreement No. 243273. This work was also supported by grants from the Biotechnology and Biological Sciences Research Council (BB/E0154761) and the Medical Research Council (G0601503). In addition, Colin J. Akerman was supported by a Fellowship from the Research Councils UK and British Pharmacological Society; Carlos D. Aizenman was supported by the National Eye Institute of the NIH, the NSF and the Klingenstein Fund; and Blake A. Richards was supported by a Welcome Trust Doctoral Fellowship and a PostGraduate Scholarship from the Natural Sciences and Engineering Research Council of Canada.

fication of neuronal networks. Nature 264, 705-712.

Cheng, H. J., Nakamoto, M., Bergemann, A. D., and Flanagan, J. G. (1995). Complementary gradients in expression and binding of ELF-1 and Mek4 in development of the topographic retinotectal projection map. Cell 82, 371-381.

Cline, H. T. (1991). Activity-dependent plasticity in the visual systems of frogs and fish. Trends Neurosci. 14, 104-111.

Cline, H. T. (2003). Sperry and Hebb: oil and vinegar? Trends Neurosci. 26, 655-661.

Cline, H. T., and Constantine-Paton, M (1989). NMDA receptor antagonists disrupt the retinotectal topographic map. Neuron 3, 413-426.

Cline, H. T., and Constantine-Paton, M. (1990). NMDA receptor agonist and antagonists alter retinal ganglion cell arbor structure in the developing frog retinotectal projection. J. Neurosci. 10, 1197-1216.
Cline, H. T., Debski, E. A., and Constantine-Paton, M. (1987). $N$-methyl-D-aspartate receptor antagonist desegregates eye-specific stripes. Proc. Natl. Acad. Sci. U.S.A. 84, 4342-4345.

Cline, H. T., Wu, G., and Malinow, R. (1996) In vivo development of neuronal structure and function. Cold Spring Harb. Symp. Quant. Biol. 61, 95-104.

Constantine-Paton, M., and Law, M. I. (1978). Eye-specific termination bands in tecta of three-eyed frogs. Science 202, 639-641.

Dahmen, J. C., Hartley, D. E. H., and King, A. J. (2008). Stimulus-timingdependent plasticity of cortical frequency representation. J. Neurosci. 28, 13629-13639.

Dan, Y., and Poo, M. (2004). Spike timingdependent plasticity of neural circuits. Neuron 44, 23-30.

Davison, A. P., and Fregnac, Y. (2006). Learning cross-modal spatial transformations through spike 
timing-dependent plasticity. $J$. Neurosci. 26, 5604-5615.

Debanne, D., Gohwiler, B. H., and Thompson, S. M. (1998). Long-term synaptic plasticity between pairs of individual CA3 pyramidal cells in rat hippocampal slice cultures. J. Physiol. (Lond.) 507, 237-247.

Debski, E. A., and Cline, H. T. (2002). Activity-dependent mapping in the retinotectal projection. Curr. Opin. Neurobiol. 12, 93-99.

Deeg, K. E., Sears, I. B., and Aizenman, C. D. (2009). Development of multisensory convergence in the Xenopus optic tectum. J. Neurophysiol. 102, 3392-3404.

Dong, W., Lee, R. H., Xu, H., Yang, S., Pratt, K. G., Cao, V., Song, Y. K., Nurmikko, A., and Aizenman, C. D. (2009). Visual avoidance in Xenopus tadpoles is correlated with the maturation of visual responses in the optic tectum. J. Neurophysiol. 101, 803-815.

Drescher, U., Kremoser, C., Handwerker, C., Löschinger, J., Noda, M., and Bonhoeffer, F. (1995). In vitro guidance of retinal ganglion cell axons by RAGS, a $25 \mathrm{kDa}$ tectal protein related to ligands for Eph receptor tyrosine kinases. Cell 82, 359-370.

Dudkin, E. A., and Gruberg, E. R. (2003). Nucleus isthmi enhances calcium influx into optic nerve fiber terminals in Rana pipiens. Brain Res. 969, 44-52.

Dunfield, D., and Haas, K. (2009). Metaplasticity governs natural experience-driven plasticity of nascent embryonic brain circuits. Neuron 64 , 240-250.

Edwards, J. A., and Cline, H. T. (1999). Light-induced calcium influx into retinal axons is regulated by presynaptic nicotinic acetylcholine receptor activity in vivo. J. Neurophysiol. 81, 895-907.

Engert, F., Tao, H. W., Zhang, L. I., and Poo, M. (2002). Moving visual stimuli rapidly induce direction sensitivity of developing tectal neurons. Nature 419 , 470-475.

Gaze, R. M. (1958). The representation of the retina on the optic lobe of the frog. Exp. Physiol. 43, 209-214.

Gaze, R. M. (1959). Regeneration of the optic nerve in Xenopus laevis. Exp. Physiol. 44, 290-308.

Gaze, R. M., Keating, M. J., and Chung, S. H. (1974). The evolution of the retinotectal map during development in Xenopus. Proc. R. Soc. Lond. B Biol. Sci. 185, 301-330.

Gaze, R. M., Keating, M. J., Ostberg, A., and Chung, S. H. (1979). The relationship between retinal and tectal growth in larval Xenopus: implications for the development of the retino-tectal projection. J. Embryol. Exp. Morphol. $53,103-143$.

Gernert, M., and Ewert, J. (1995). Cholinergic, GABAergic, and dopaminergic influences on visually evoked field potentials in the superficial optic tectum of Bufo marinus. Comp. Biochem. Physiol. A Physiol. 112, 387-401.

Grüsser, O. J., and Grüsser-Cornehls, U. (1976). "Neurophysiology of the anuran visual system," in Frog Neurobiology: A Handbook. R. Llinás and W. Precht, eds (New York: Springer-Verlag), 297-385.

Haas, K., Li, J., and Cline, H. T. (2006). AMPA receptors regulate experiencedependent dendritic arbor growth in vivo. Proc. Natl. Acad. Sci. U.S.A. 103, 12127-12131.

Hickmott, P. W., and Constantine-Paton, M. (1993). The contributions of NMDA, non-NMDA, and GABA receptors to postsynaptic responses in neurons of the optic tectum. J. Neurosci. 13, 4339-4353.

Hiramoto, M., and Cline, H. T. (2009). Convergence of multisensory inputs in Xenopus tadpole tectum. Dev. Neurobiol. 69, 959-971.

Holt, C. E. (1984). Does timing of axon outgrowth influence initial retinotectal topography in Xenopus? J. Neurosci. 4, 1130-1152.

Holt, C. E., and Harris, W. A. (1993). Position, guidance, and mapping in the developing visual system. $J$. Neurobiol. 24, 1400-1422.

Holt, C. E., and Harris, W. A. (1983). Order in the initial retinotectal map in Xenopus: a new technique for labelling growing nerve fibres. Nature 301, 150-152.

Huo, J., Zhijun, Y., and Murray, A. (2008). "Modeling visual and auditory integration of barn owl superior colliculus with STDP," in Proceedings of IEEE Conference on Cybernetics and Intelligent Systems (Chengdu: IEEE), 1124-1128.

Ingle, D. (1973a). Evolutionary perspectives on the function of the optic tectum. Brain Behav. Evol. 8, 211-237.

Ingle, D. (1973b). Two visual systems in the frog. Science 181, 1053-1055.

Isa, T. (2002). Intrinsic processing in the mammalian superior colliculus. Curr. Opin. Neurobiol. 12, 668-677.

Jacobson, M. (1968). Development of neuronal specificity in retinal ganglion cells of Xenopus. Dev. Biol. 17, 202-218.

Kampa, B. M., Letzkus, J. J., and Stuart, G. J. (2007). Dendritic mechanisms controlling spike-timing-dependent synaptic plasticity. Trends Neurosci. $30,456-463$

Keating, M. J., and Feldman, J. D. (1975). Visual deprivation and intertectal neuronal connexions in Xenopus laevis. Proc. R. Soc. Lond. B Biol. Sci. 191, 467-474.

Lázár, G. (1973). The development of the optic tectum in Xenopus laevis: a Golgi study. J. Anat. 116, 347.

Lee, P. H., Helms, M. C., Augustine, G. J., and Hall, W. C. (1997). Role of intrinsic synaptic circuitry in collicular sensorimotor integration. Proc. R. Soc. Lond. B Biol. Sci. 94, 13299-13304.

Levy, W. B., and Steward, O. (1983) Temporal contiguity requirements for long-term associative potentiation/depression in the hippocampus. Neuroscience 8, 791-797.

Lien, C., Mu, Y., Vargas-Caballero, M., and Poo, M. (2006).Visual stimuli-induced LTD of GABAergic synapses mediated by presynaptic NMDA receptors. Nat. Neurosci. 9, 372-380.

Li, Y., Fitzpatrick, D., and White, L. E. (2006). The development of direction selectivity in ferret visual cortex requires early visual experience. Nat. Neurosci. 9, 676-681.

Li, Y., Van Hooser, S. D., Mazurek, M., White, L. E., and Fitzpatrick, D. (2008). Experience with moving visual stimuli drives the early development of cortical direction selectivity. Nature 456, 952-956.

Liu, Y., Zhang, L. I., and Tao, H. W. (2007). Heterosynaptic scaling of developing GABAergic synapses: dependence on glutamatergic input and developmental stage. J. Neurosci. 27, 5301-5312.

Maass, W., and Markram, H. (2004). On the computational power of circuits of spiking neurons. J. Comp. Syst. Sci. 69, 593-616.

Mann, F., Ray, S., Harris, W. A., and Holt, C. E. (2002). Topographic mapping in dorsoventral axis of the Xenopus retinotectal system depends on signaling through ephrin-B ligands. Neuron 35, 461-473.

Markram, H., Lubke, J., Frotscher, M., and Sakmann, B. (1997). Regulation of synaptic efficacy by coincidence of postsynaptic APs and EPSPs. Science 275, 213-215.

Martin, S. J., Grimwood, P.D., and Morris, R. G. M. (2000). Synaptic plasticity and memory: an evaluation of the hypothesis. Annu. Rev. Neurosci. 23, 649-711.

McCormick, D. A., Shu, Y., Hasenstaub, A., Sanchez-Vives, M., Badoual, M., and Bal, T. (2003). Persistent cortical activity: mechanisms of generation and effects on neuronal excitability. Cereb. Cortex 13, 1219-1231.

Mehta, M. R., Quirk, M. C., and Wilson, M. A. (2000). Experience-dependent asymmetric shape of hippocampal receptive fields. Neuron 25, 707-715.

Meliza, C. D., and Dan, Y. (2006) Receptive-field modification in rat visual cortex induced by paired visual stimulation and single-cell spiking. Neuron 49, 183-189.

Meredith, R. M., Floyer-Lea, A. M., and Paulsen, O. (2003). Maturation of long-term potentiation induction rules in rodent hippocampus: role of GABAergic inhibition. J. Neurosci. 23 11142-11146.

Meyer, R. L. (1983). Tetrodotoxin inhibits the formation of refined retinotopography in goldfish. Dev. Brain Res. 6, 293-298.

Mu, Y., and Poo, M. (2006). Spike timingdependent LTP/LTD mediates visual experience-dependent plasticity in a developing retinotectal system. Neuron 50, 115-125.

Nakagawa, H., Miyazaki, H., and Matsumoto, N. (1997). Principal neuronal organization in the frog optic tectum revealed by a current source density analysis. Vis. Neurosci. 14, 263-275.

Niell, C. M., and Smith, S. J. (2005). Functional imaging reveals rapid development of visual response properties in the zebrafish tectum. Neuron $45,941-951$.

Nieuwkoop, P. D., and Faber, J. (1967). Normal Table of Xenopus laevis (Daudin): A Systematical and Chronological Survey of the Development from the Fertilized Egg Till the End of Metamorphosis. Amsterdam: North-Holland Publishing Co.

Nikundiwe, A. M. and Nieuwenhuys, R. (1983). The cell masses in the brainstem of African clawed frog Xenopus Laevis: a topographical and topological analysis. J. Comp. Neurol. 213, 199-219.

O'Rourke, N. A., Cline, H. T., and Fraser, S. E. (1994). Rapid remodeling of retinal arbors in the tectum with and without blockade of synaptic transmission. Neuron 12, 921-934.

O'Rourke, N. A., and Fraser, S. E. (1986). Dynamic aspects of retinotectal map formation revealed by a vital-dye fiber-tracing technique. Dev. Biol. 114, 265-276

Pratt, K. G., and Aizenman, C. D. (2007). Homeostatic regulation of intrinsic excitability and synaptic transmission in a developing visual circuit. $J$. Neurosci. 27, 8268-8277.

Pratt, K. G., and Aizenman, C. D. (2009). Multisensory integration in mesencephalic trigeminal neurons in Xenopus tadpoles.J. Neurophysiol. 102, 399-412.

Pratt, K. G., Dong, W., and Aizenman, C. D. (2008). Development and spike timing-dependent plasticity of recurrent excitation in the Xenopus optic tectum. Nat. Neurosci. 11, 467-475.

Rajan,I., and Cline,H.T.(1998). Glutamate receptor activity is required for normal 
development of tectal cell dendrites in vivo. J. Neurosci. 18, 7836-7846.

Ramdya, P., and Engert, F. (2008). Emergence of binocular functional properties in a monocular neural circuit. Nat. Neurosci. 11, 1083-1090.

Rao, R. P. N., and Sejnowski, T. J. (2001). "Predictive learning of temporal sequences in recurrent neocortical circuits," in Novartis Foundation Symposia, 208-229. Available at: http://www.scopus. com/inward/record.url?eid $=2$-s2.00035235894\&partnerID $=40$.

Reh, T. A., and Constantine-Paton, M. (1984). Retinal ganglion cell terminals change their projection sites during larval development of Rana pipiens. J. Neurosci. 4, 442-457.

Reh, T. A., and Constantine-Paton, M. (1985). Eye-specific segregation requires neural activity in threeeyed Rana pipiens. J. Neurosci. 5, 1132-1143.

Ruthazer, E. S., Akerman, C. J., and Cline, H. T. (2003). Control of axon branch dynamics by correlated activity in vivo. Science 301, 66-70.

Ruthazer, E. S., and Cline, H. T. (2004). Insights into activity-dependent map formation from the retinotectal system: a middle-of-the-brain perspective. J. Neurobiol. 59, 134-146.

Saito, Y., and Isa, T. (2003). Local excitatory network and NMDA receptor activation generate a synchronous and bursting command from the superior colliculus. J. Neurosci. 23, 5854-5864.

Sakaguchi, D. S., and Murphey, R. K. (1985). Map formation in the developing Xenopus retinotectal system: an examination of ganglion cell terminal arborizations. J. Neurosci. 5, 3228-3245.

Scanziani, M., and Hausser, M. (2009). Electrophysiology in the age of light. Nature 461, 930-939.

Schmidt, J. T. (1985). Formation of retinotopic connections: Selective stabilization by an activity-dependent mechanism. Cell. Mol. Neurobiol. 5, 65-84.

Schmidt, J. T., and Edwards, D. L. (1983). Activity sharpens the map during the regeneration of the retinotectal projection in goldfish. Brain Res. 269, 29-39.

Shen, W., Da Silva, J. S., He, H., and Cline, H. T. (2009). Type A GABA-receptordependent synaptic transmission sculpts dendritic arbor structure in Xenopus tadpoles in vivo. J. Neurosci. 29, 5032-5043.

Shon, A. P., Rao, R. P. N., and Sejnowski, T.J. (2004). Motion detection and prediction through spike-timing dependent plasticity. Network 15, 179-198.

Sjöström, P. J., Turrigiano, G. G., and Nelson, S. B. (2001). Rate, timing, and cooperativity jointly determine cortical synaptic plasticity. Neuron 32, 1149-1164.

Sparks, D. L. (1986). Translation of sensory signals into commands for control of saccadic eye movements: role of primate superior colliculus. Physiol. Rev. 66, 118-171.

Sperry, R.W. (1944). Optic nerve regeneration with return of vision in anurans. J. Neurophysiol. 7, 57-69.

Sperry, R.W. (1963). Chemoaffinity in the orderly growth of nerve fiber patterns and connections. Proc. Natl. Acad. Sci. U.S.A. 50, 703-710.

Székely, G., and Lázár, G. (1967). Golgi studies on the optic center of the frog. J. Hirnforsch. 9, 329-344.

Székely, G., and Lázár, G. (1976). "Cellular and synaptic architecture of the optic tectum," in Frog Neurobiology: a Handbook, R. Llinás and W. Precht, eds. (Berlin: Springer-Verlag), 407-434.

Tao, H. W., and Poo, M. (2005). Activitydependent matching of excitatory and inhibitory inputs during refinement of visual receptive fields. Neuron 45 , 829-836.

Tao, H. W., Zhang, L. I., Engert, F., and Poo, M. (2001). Emergence of input specificity of LTP during development of retinotectal connections in vivo. Neuron 31, 569-580.

Tremblay, M., Fugère, V., Tsui, J., Schohl, A., Tavakoli, A., Travençolo, B. A., Costa, L. F., and Ruthazer, E. S. (2009). Regulation of radial glial motility by visual experience. J. Neurosci. 29 14066-14076.

Tzounopoulos, T., Kim, Y., Oertel, D., and Trussell, L. O. (2004). Cell-specific, spike timing-dependent plasticities in the dorsal cochlear nucleus. Nat. Neurosci. 7, 719-725.

Udin, S. B. (1985). The role of visual experience in the formation of binocular projections in frogs. Cell. $\mathrm{Mol}$. Neurobiol. 5, 85-102.

Udin, S. B. (2007). The instructive role of binocular vision in the Xenopus tectum. Biol. Cybern. 97, 493-503.

Udin, S. B., and Fisher, M. D. (1985). The development of the Nucleus Isthmi in Xenopus laevis. I. Cell genesis and the formation of connections with the tectum. J. Comp. Neurol. 232, 25-35.

Udin, S. B., and Keating, M. J. (1981) Plasticity in a central nervous pathway in Xenopus: anatomical changes in the isthmotectal projection after larval eye rotation. J. Comp. Neurol. 203, 575-594.

VanRullen, R., Guyonneau, R., and Thorpe, S. (2005). Spike times make sense. Trends Neurosci. 28, 1-4.

Vislay-Meltzer, R. L., Kampff, A. R., and Engert, F. (2006). Spatiotemporal specificity of neuronal activity directs the modification of receptive fields in the developing retinotectal system. Neuron 50, 101-114.

Whitelaw, V. A., and Cowan, J. D. (1981) Specificity and plasticity of retinotectal connections: a computational model. J. Neurosci. 1, 1369-1387.

Willshaw, D. J., and Von Der Malsburg, C. (1976). How patterned neural connections can be set up by self-organization. Proc. R. Soc. Lond. B Biol. Sci. 194, 431-445.
Wu, G., and Cline, H. T. (1998). Stabilization of dendritic arbor structure in vivo by CaMKII. Science 279, 222-226.

Yao, H., and Dan, Y. (2001). Stimulus timing-dependent plasticity in cortical processing of orientation. Neuron 32, 315-323.

Yu, L., Stein, B. E., and Rowland, B. A. (2009). Adult plasticity in multisensory neurons: short-term experience-dependent changes in the superior colliculus. J. Neurosci. 29, 15910-15922.

Zhang, L. I., Tao, H. W., Holt, C. E. Harris, W. A., and Poo, M. (1998). A critical window for cooperation and competition among developing retinotectal synapses. Nature 395, 37-44.

Zhang, L. I., Tao, H. W., and Poo, M. (2000). Visual input induces longterm potentiation of developing retinotectal synapses. Nat. Neurosci. 3, 708-715.

Conflict of Interest Statement: The authors declare that the research was conducted in the absence of any commercial or financial relationships that could be construed as a potential conflict of interest.

Received: 08 February 2010; paper pending published: 16 February 2010; accepted: 17 May 2010; published online: 10 June 2010.

Citation: Richards BA, Aizenman CD and Akerman CJ (2010) In vivo spike-timingdependent plasticity in the optic tectum of Xenopus laevis. Front. Syn. Neurosci. 2:7. doi: 10.3389/fnsyn.2010.00007

Copyright (C) 2010 Richards, Aizenman and Akerman. This is an open-access article subject to an exclusive license agreement between the authors and the Frontiers Research Foundation, which permits unrestricted use, distribution, and reproduction in any medium, provided the original authors and source are credited. 